\title{
Hypotensive effect of atorvastatin in hypertensive patients: the association among flow-mediated dilation, oxidative stress and endothelial dysfunction
}

Agnieszka M. Tycinska ${ }^{1}$, Jacek Janica², Barbara Mroczko ${ }^{3}$, Wlodzimierz J. Musiall, Robert Sawicki', Bozena Sobkowicz', Karol Kaminski', Urszula Lebkowska², Maciej Szmitkowski ${ }^{3}$

1Department of Cardiology, Medical University of Bialystok, Poland 2Department of Radiology, Medical University of Bialystok, Poland

${ }^{3}$ Department of Biochemical Diagnostics, Medical University of Bialystok, Poland

Submitted: 29 March 2011

Accepted: 19 August 2011

Arch Med Sci 2011; 7, 6: 955-962

DOI: 10.5114/AOMS.2011.26606

Copyright @ 2011 Termedia \& Banach

\section{Abstract}

Introduction: To investigate the hypothesis that atorvastatin decreases blood pressure (BP) values and improves endothelial function assessed by flow-mediated dilation (FMD) in normolipidaemic hypertensive patients.

Material and methods: Fifty-six hypertensive patients were randomized in a 2 : 1 proportion to atorvastatin ( $80 \mathrm{mg} /$ day/3 months; group $\mathrm{A} ; n=39$ ) or previous standard anti-hypertensive therapy (group B), which means the patients were treated with angiotensin-converting enzyme inhibitors, diuretics, $\beta$-blockers, calcium antagonists and angiotensin receptor blockers. The study had a crossover design: after 3 months, both groups were changed (group $A^{*}$ stopped and group $B^{*}$ started atorvastatin treatment). Nitric oxide (NO), total antioxidant status (TAS), endothelin-1 (ET-1), and peroxide concentrations as well as FMD were measured before, after 3 and after 6 months of treatment. Atorvastatin added to existing treatment decreased BP in both groups.

Results: Flow-mediated dilation improved in both statin-treated groups, but only significantly in group $B^{*}$ (from $11.9 \pm 8.3 \%$ to $22.1 \pm 9.0 \%$; $p<0.05$ ). In patients with FMD improvement, there was a greater BP reduction. After treatment discontinuation, FMD significantly decreased (from $19.6 \pm 12.6 \%$ to 13.0 $\pm 10.5 \%$; $p<0.05$ ), which was consistent with BP increase. Changes in FMD were not significantly related to the increase in NO and TAS concentrations and decrease in ET-1 and peroxides measurements.

Conclusions: The hypotensive effect of atorvastatin is associated with FMD improvement in normolipidaemic, hypertensive patients. Although this could be related to changes in oxidative stress and endothelial function, this was not demonstrated in this study and warrants further investigation.

Key words: arterial hypertension, endothelin-1, flow-mediated dilation, nitric oxide, peroxides, total antioxidant status.

\section{Introduction}

Essential arterial hypertension and hyperlipidaemia are powerful risk factors of cardiovascular disease. Endothelial dysfunction is a leading cause of early development and preservation of arterial hypertension [1]. Endothelial function can be assessed using ultrasound methods to observe the arterial flow-mediated dilation (FMD). It is also possible to assess bio-

\author{
Corresponding author: \\ Agnieszka M. Tycinska \\ $\mathrm{MD}, \mathrm{PhD}$ \\ Department of Cardiology \\ Medical University \\ of Bialystok \\ 24a Sklodowskiej-Curie \\ 15-276 Bialystok, Poland \\ Phone: +48 857468656 \\ Fax: +48 857468604 \\ E-mail: \\ agnieszka.tycinska@gmail.com
}


markers of endothelial function, of which nitric oxide (NO) and endothelin-1 (ET-1) play a crucial role. The increase in ET-1, as well as NO defective endothelial release, is common in hypertensive patients [1, 2]. Moreover, it is thought that high blood pressure values are associated with a loss of balance between the status of oxidative stress and the level of antioxidants [3].

Apart from lowering cholesterol, statins demonstrate other biological actions, including anti-inflammatory effects and improved endothelial function. Current data indicate that statins could have an extensive range of indications [4].

Statins interact with blood pressure control in different populations of hypertensive patients [5]. However, the influence of statin therapy on peroxide concentrations as well as total antioxidant status in normolipidaemic hypertensive patients remains unclear.

This study sought to investigate, in a crossover design, the improvement of endothelial dysfunction in normolipidaemic patients with hypertension under treatment, before and after atorvastatin treatment, and to determine whether the alteration of biomarkers assessing endothelial function (NO and ET-1), oxidative stress (measured by Oxystat), or total antioxidative status could be responsible for that effect.

\section{Material and methods}

\section{Study population}

Among 92 patients referred to our outpatient clinic with previously diagnosed and treated essential arterial hypertension, we studied 56 (32 males; aged 44 to 64 years) non-smoking, normolipidaemic ones. The mean time of a history of arterial hypertension was $5 \pm 3.2$ years. Patients were randomized in an open-label manner in a $2: 1$ proportion to atorvastatin ( $80 \mathrm{mg} /$ day/3 months; group A, $n=39$ ), or to the standard, previous therapy (group $\mathrm{B}, n=17$ ), which means that these patients were treated with standard anti-hypertensive agents including angiotensin-converting enzyme inhibitors (ACE-I), diuretics, $\beta$-blockers (BB), calcium antagonists (CA) and angiotensin receptor blockers (ARB). The proportion of anti-hypertensive agents was similar between groups. The exact method of randomization and the percentage of antihypertensive agents are described elsewhere [6]. The mean value of total cholesterol for the whole group was $185.2 \mathrm{mg} / \mathrm{dl}$ (SD \pm 38.8$)$. Atorvastatin significantly reduced total cholesterol (TC), low density lipoprotein (LDL) and triglyceride (TG) concentrations [6]. The activities of alanine and aspartate aminotransferases did not significantly change after atorvastatin treatment.

The study was done in a crossover design - after 3 months, the groups were changed: group $A^{*}$ and
$B^{\star}$. With this type of study, every patient serves as his or her own control. Blood pressure were measured using a 24-h ambulatory blood pressure measurement device (ABPM, Tracker Reynolds NIBP2, Reynolds Medical, Hertford, UK) as previously described [6]. Basic mean values for systolic and diastolic blood pressures were similar in groups A and B: systolic blood pressure $129 \pm 11 \mathrm{mmHg}$ vs. $129.5 \pm 13 \mathrm{mmHg}$ and diastolic blood pressure $76 \pm 9 \mathrm{mmHg}$ vs. $74 \pm 7.6 \mathrm{mmHg}(p=\mathrm{NS})$.

The study design complied with the Helsinki Declaration of 1975 (revised in 1996), and it was approved by the local institutional committee on human research (Institutional Review Board - Local Bioethics Committee of Bialystok Medical University). Informed consent of all participants covered by the study was obtained.

Endothelium-dependent FMD was estimated following the instructions given by Corretti and associates [7]. Flow-mediated dilation was determined in both groups at baseline, after 3 months (before crossover), and at the end of the study ( 3 months after crossover). All participants fasted for $12 \mathrm{~h}$ and avoided exercise for 4 to $6 \mathrm{~h}$ before FMD examination.

The brachial artery diameter was measured $6 \mathrm{~cm}$ above the antecubital space using a high-resolution ultrasound $7.5-\mathrm{MHz}$ linear array transducer (Toshiba SSA-140A). Baseline imaging was followed by 5 -min occlusion of arterial flow, achieved by inflating a pneumatic cuff above the antecubital fossa (upper arm occlusion to at least $50 \mathrm{mmHg}$ above systolic blood pressure to occlude arterial flow). After deflating the pneumatic cuff, the brachial artery was imaged continuously for $3 \mathrm{~min}$ (reactive hyperaemia and endothelium-dependent dilation). The internal diameter (measured in $\mathrm{mm}$ ) was defined as the distance between the intimalumen interface of the near wall and the intimalumen interface of the far wall, and was assessed during late-diastole corresponding to the $\mathrm{R}$ wave of the electrocardiogram (ECG) trace. The maximum diameter was taken into consideration. Flow mediated dilation was expressed as percentage change from rest $[\times 100$ (brachial artery diameter at peak hyperaemia - diameter at rest)/diameter at rest]. Measurements were performed in a blinded manner, without knowledge of the patient's group assignment.

\section{Blood sampling and biochemical measurements}

Venous blood samples were obtained from fasting patients between 8:00 am and 10:00 am. The patients were lying comfortably in the supine position for $15 \mathrm{~min}$. After that time, an antecubital vein of the non-dominant forearm was cannulated, and after another $20 \mathrm{~min}$, venous blood samples for 
total antioxidant status, peroxides, NO, and ET-1 assays were collected into tubes with the clotting activation system (tubes for ET-1 were put on ice immediately after collection). All samples were centrifuged within $2 \mathrm{~h}$ after drawing and stored at $-80^{\circ} \mathrm{C}$ until assayed.

Serum concentration of total antioxidant status was assayed using an enzymatic method with peroxidase by commercially available RANDOX total antioxidant status kits (Randox, Ardmore, United Kingdom) according to the manufacturer's instructions. This method has been described previously [8].

Plasma concentrations of peroxides were measured using Oxystat colorimetric assay kits (Biomedica, Wien, Austria). The results show a direct correlation between free radicals and circulating biological peroxides, and thus allow characterization of the oxidative status in biological samples. The peroxide concentration is determined by the reaction of the biological peroxides with peroxidase and subsequent colour-reaction using tetramethylbenzidine as a substrate. After addition of a stop solution, the coloured liquid is measured photometrically at $450 \mathrm{~nm}$ [9]. The intra-assay coefficient of variation is referred to by the manufacturer of assay kits as $3.1 \%$ at peroxide mean concentration of $221 \mu \mathrm{mol} / \mathrm{l}, \mathrm{SD}=6.9 \mu \mathrm{mol} / \mathrm{l}$.

Serum levels of NO were measured using colorimetric total NO/nitrite/nitrate assay kits (R\&D Systems, Abingdon, England) [6]. The intra-assay CV\% is reported by the manufacturer of assay kits as $2.5 \%$ at $\mathrm{NO}$ mean concentration of $30 \mu \mathrm{mol}$, $\mathrm{SD}=0.76 \mathrm{U} / \mathrm{l}$.

Serum concentrations of ET-1 were measured using enzyme-linked immunosorbent assay (ELISA) kits (Biomedica, Wien, Austria). The intra-assay coefficient of variation is referred to by the manufacturer of assay kits as 4\% at ET-1 mean concentration of $2.02 \mathrm{fmol} / \mathrm{ml}, \mathrm{SD}=0.08$.

\section{Statistical analysis}

Results are expressed as means \pm standard deviation (SD) or $95 \%$ confidence interval, $\mathrm{Cl}$ (variables with the normal distribution) or $n$, percentage, depending on the type of variables. Particular clinical and biochemical parameters were compared using ANOVA for repeated measures for principal factors or recurrent measurements and a $\chi^{2}$ test. All analyses were done using Statistica 9.1 PL. Value of $p$ below 0.05 was considered statistically significant.

\section{Results}

Baseline clinical and biochemical characteristics of the study patients are shown in Table I. Atorvastatin, added to the existing therapy for 3 months, decreased blood pressure values in both groups - in group A: systolic blood pressure -
Table I. Baseline clinical and biochemical characteristics of study patients

\begin{tabular}{|lccc|}
\hline Parameter & $\begin{array}{c}\text { Group A } \\
(n=39)\end{array}$ & $\begin{array}{c}\text { Group B } \\
(n=17)\end{array}$ & $\begin{array}{c}\text { Value } \\
\text { of } p\end{array}$ \\
\hline $\begin{array}{l}\text { Age, mean } \pm \text { SD } \\
\text { [years] }\end{array}$ & $52.5 \pm 14.7$ & $47.9 \pm 18.1$ & 0.31 \\
\hline Sex, males [\%] & 43 & 53 & 0.49 \\
\hline $\begin{array}{l}\text { TAS, mean } \pm \text { SD } \\
\text { [mmol/l] }\end{array}$ & $1.5 \pm 0.2$ & $1.4 \pm 0.3$ & 0.9 \\
\hline $\begin{array}{l}\text { Peroxides, } \\
\text { mean } \pm \text { SD [ } \mu \text { mol/l] }\end{array}$ & $326.8 \pm 194.3$ & $249.1 \pm 139.8$ & 0.05 \\
\hline $\begin{array}{l}\text { NO, mean } \pm \text { SD } \\
{[I U / l]}\end{array}$ & $22.6 \pm 14.2$ & $39.9 \pm 29.5$ & 0.4 \\
\hline $\begin{array}{l}\text { ET-1, mean } \pm \text { SD } \\
{[\text { fmol/ml] }}\end{array}$ & $1.32 \pm 2.2$ & $0.5 \pm 0.2$ & 0.14 \\
\hline
\end{tabular}

SD - standard deviation, TAS - total antioxidant status, NO - nitric oxide, ET-1 - endothelin-1

$5.7 \mathrm{~mm} \mathrm{Hg}$ (95\% confidence interval (Cl) $-4.1 \mathrm{mmHg}$ to $-7.2 \mathrm{mmHg}$ ) and diastolic blood pressure $3.9 \mathrm{mmHg}(95 \% \mathrm{Cl}-2.7 \mathrm{mmHg}$ to $-5.0 \mathrm{mmHg})$, and in group $\mathrm{B}^{*}$ : systolic blood pressure $-1.23 \mathrm{mmHg}$ (95\% Cl -6.93 to 4.46$)$ and diastolic blood pressure $-2.0 \mathrm{mmHg}(95 \% \mathrm{Cl}-5.46$ to 1.46$)$ (Figure 1).

Flow-mediated dilation showed significant improvement in group B* (from $11.9 \pm 8.3 \%$ to 22.1 $\pm 9.0 \% ; p<0.05$ ), but was not significant in group $A$ (from $18.7 \pm 8.9 \%$ to $19.6 \pm 12.6 \% ; p=0.7$ ) (Figure 2). However, in group $A^{*}$ (after crossover), systolic blood pressure values significantly increased $(p=0.02)$ and FMD significantly decreased (from $19.6 \pm 12.6 \%$ to $13.0 \pm 10.5 \%$; $p<0.05$ ) (Figures 1 and 2 ).

In an ANOVA test, in patients with FMD improvement after atorvastatin treatment, there was a trend to a greater blood pressure reduction in group A (before crossover): systolic blood pressure $-7.2 \mathrm{mmHg}(95 \% \mathrm{Cl}-10.75$ to -3.62$)$ and diastolic blood pressure $-4.8 \mathrm{mmHg}(95 \% \mathrm{Cl}-6.85$ to 2.78 ) and a significant blood pressure reduction in group $B^{*}$ (after crossover): systolic blood pressure $-3.1 \mathrm{mmHg}(95 \% \mathrm{Cl}-9.91$ to -3.75$)$ and diastolic blood pressure $-2.4 \mathrm{mmHg}(95 \% \mathrm{Cl}-6.68$ to -1.91$)$ (Figure 3).

In group A, FMD improvement and aggravation were not associated with significant changes (the increase) in total antioxidant status concentrations: mean $0.04 \mathrm{mmol} / \mathrm{l}(95 \% \mathrm{Cl}-0.91$ to 0.18$)$ and 0.02 $\mathrm{mmol} / \mathrm{l}(95 \% \mathrm{Cl}-0.10$ to 0.13$)$ (Figure 4). Also, in that group of patients, neither FMD improvement nor impairment was associated with significant changes in Oxystat (decrease: mean -64.1 $\mu \mathrm{mol} / \mathrm{l}$ $(95 \% \mathrm{Cl}-183.9$ to 55.7$)$ and increase: mean $9.6 \mu \mathrm{mol} / \mathrm{l}(95 \% \mathrm{Cl}-103.8$ to 123.0$)$, respectively) (Figure 4).

In group $B^{*}$ (receiving statin after crossover), patients with FMD improvement had a trend to an increase in NO concentrations (mean $2.7 \mathrm{IU} / \mathrm{l}$ 

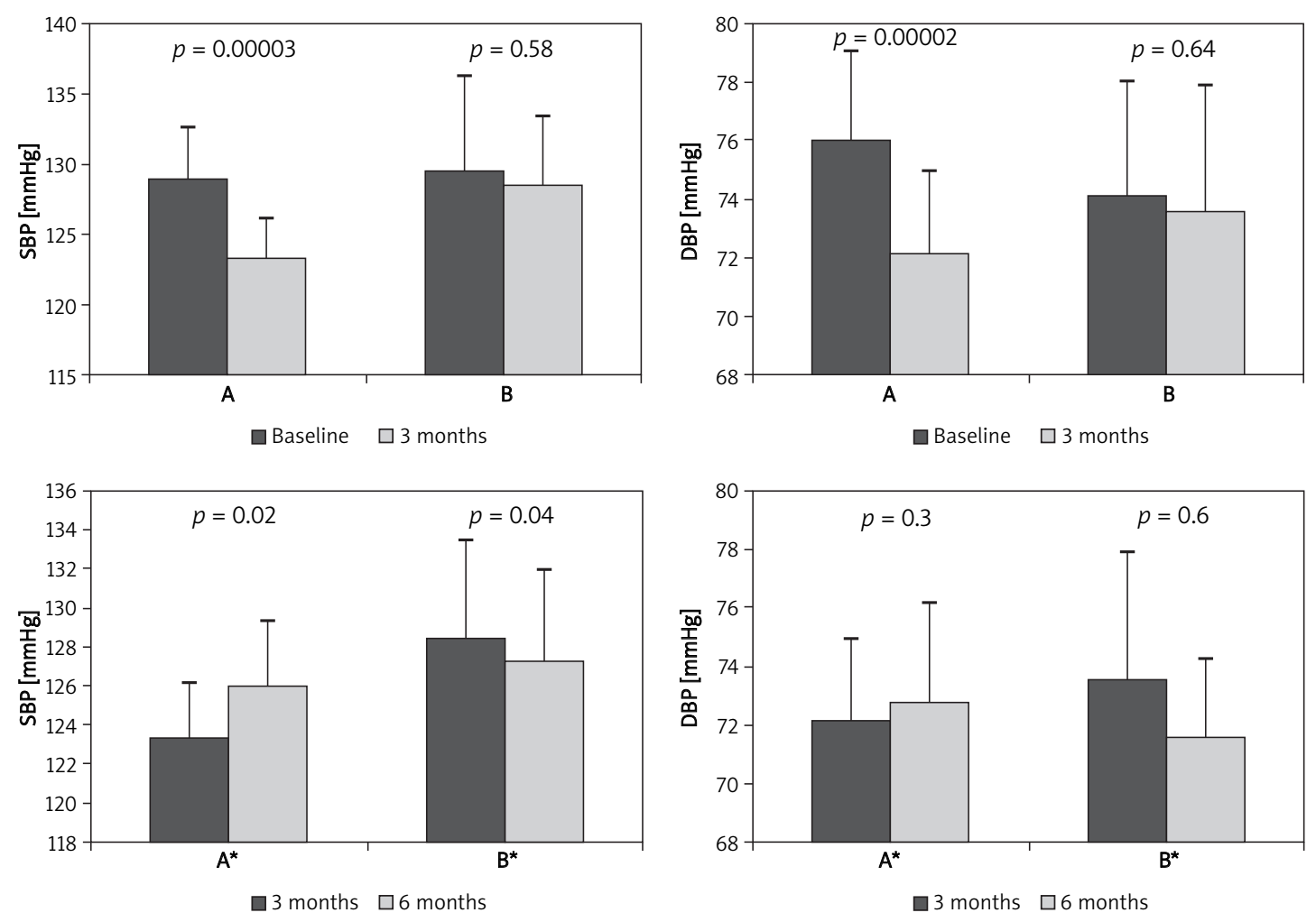

Figure 1. Changes in systolic and diastolic blood pressure values in groups $A-A^{*}$ and $B-B^{*}$ $S B P$ - systolic blood pressure, DBP - diastolic blood pressure
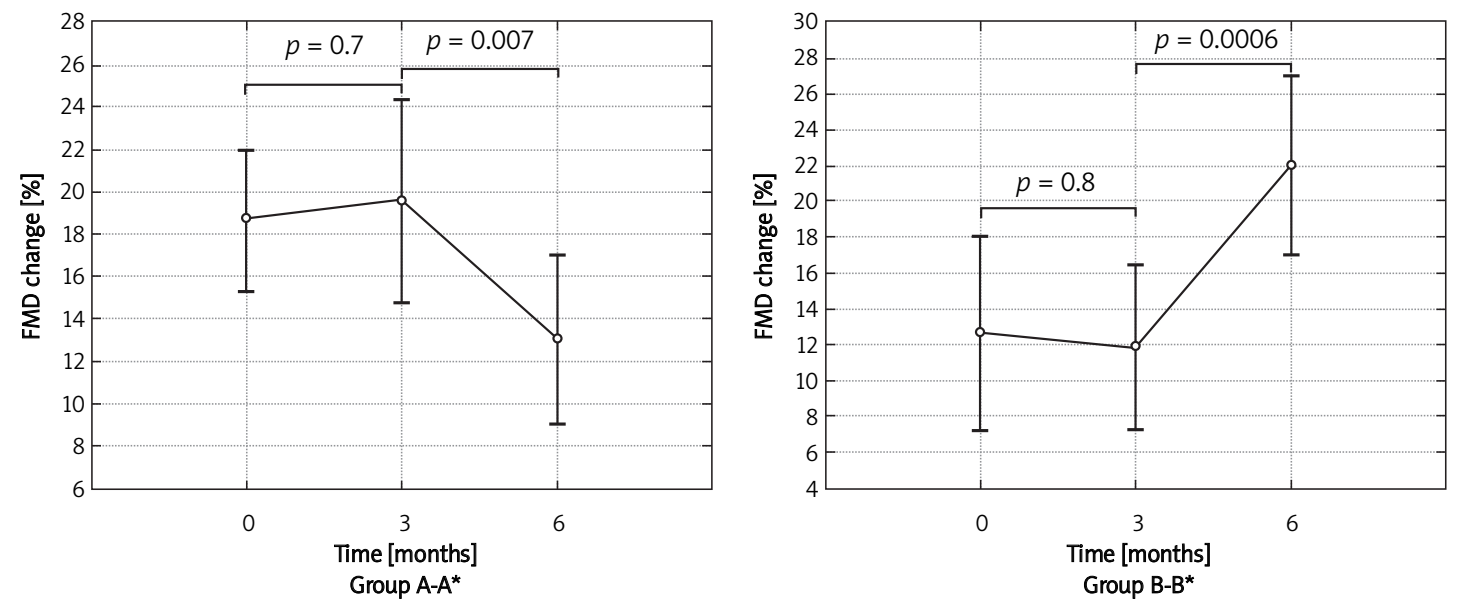

Figure 2. Flow-mediated dilation changes in both groups during the study FMD - flow-mediated dilation

(95\% Cl -5.34 to 10.91$))$ and decrease in ET-1 values (mean $-0.2 \mathrm{fmol} / \mathrm{ml}$ [ $95 \% \mathrm{Cl}-0.41$ to 0.06$]$ ) (Figure 5). In patients without FMD improvement, NO decreased (mean $-17.0 \mathrm{IU} / \mathrm{I}[95 \% \mathrm{Cl}-46.30$ to 12.26$]$ ), whereas ET-1 increased (mean $0.02 \mathrm{fmol} / \mathrm{ml}$ ( $95 \% \mathrm{Cl}-0.82$ to 0.86$)$ ) (Figure 5). However, these trends were not significant.

\section{Discussion}

The results of this study suggest that the hypotensive effect of atorvastatin is related to FMD improvement in a selected group of hypertensive patients. Two actions could be responsible for this antihypertensive effect: FMD variation probably depends on concentration changes in oxidative stress/antioxidants as well as changes in biomarkers of endothelial function (NO, ET-1).

Data have emerged that endothelial dysfunction is an early step in developing atherosclerosis and is characterized mainly by a reduction in the bioavailability of NO [10]. Endothelial function is often quantified by FMD, which represents the 


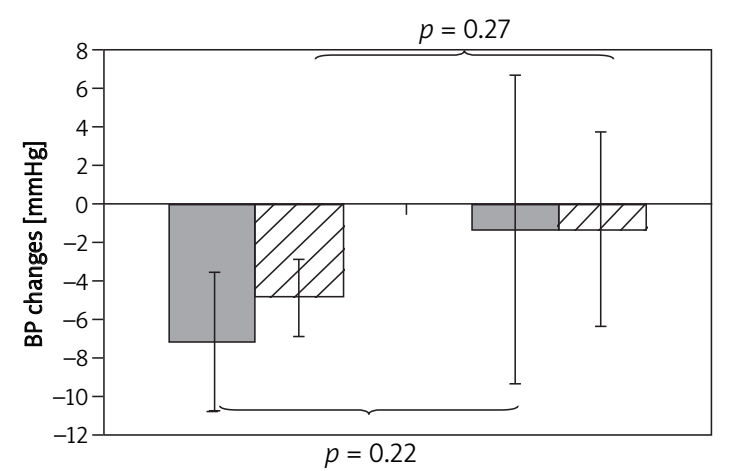

A

3 months, before crossover

$\square \mathrm{SBP} \quad \mathrm{DDBP}$

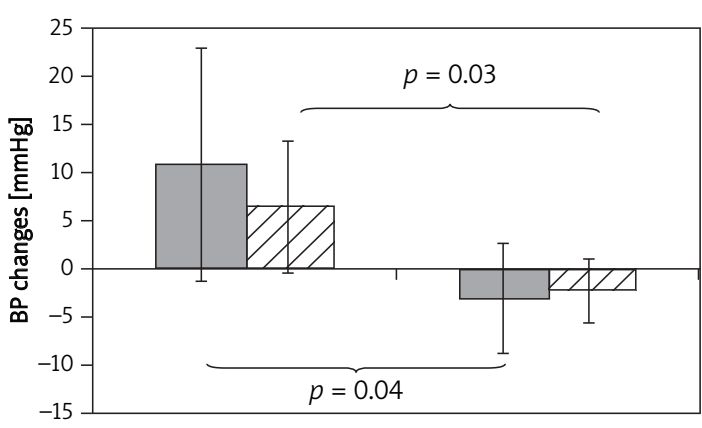

$A^{*}$

6 months, after crossover

$\square \mathrm{SBP} \quad \square \mathrm{DBP}$

Figure 3. Changes in SBP and DBP values in patients with FMD improvement (ANOVA test) $S B P$ - systolic blood pressure, DBP - diastolic blood pressure, FMD - flow-mediated dilation

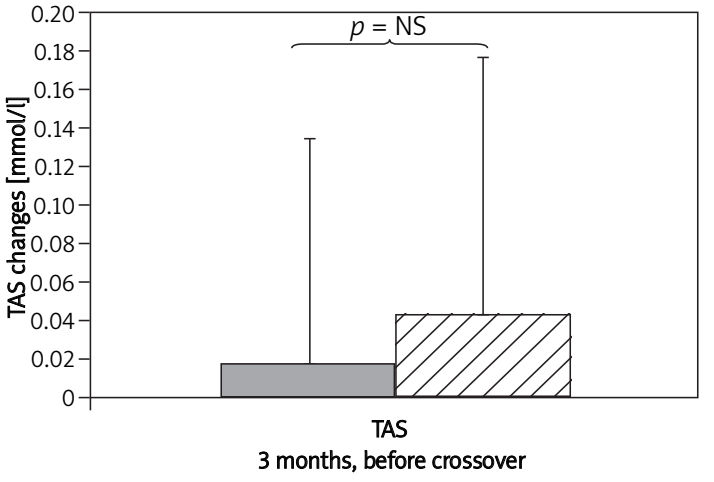

$\square$ FMD decrease $\square \neg$ FMD increase

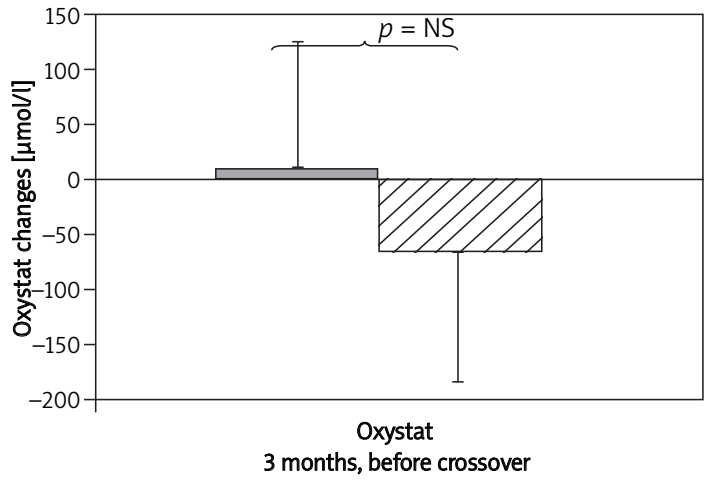

$\square$ FMD decrease $\boxminus$ FMD increase

Figure 4. FMD changes in relation to TAS and Oxystat concentrations in group A patients (all $p=\mathrm{NS}$ ) FMD - flow-mediated dilation, TAS - total antioxidant status, Oxystat - a method to measure peroxide concentrations

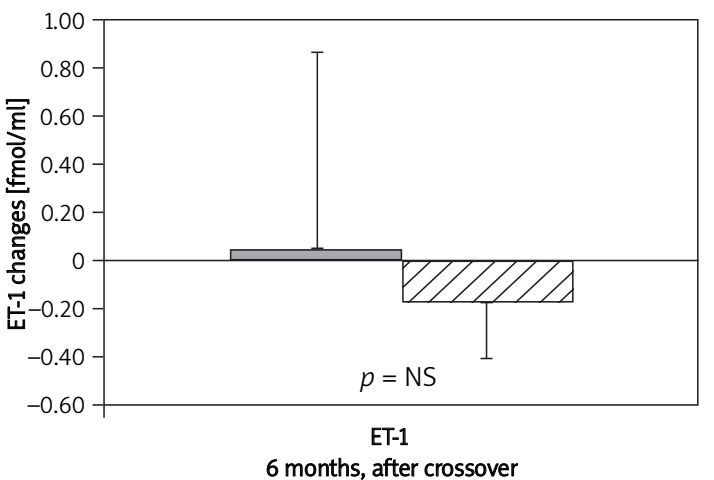

$\square$ FMD decrease $\quad \square$ FMD increase

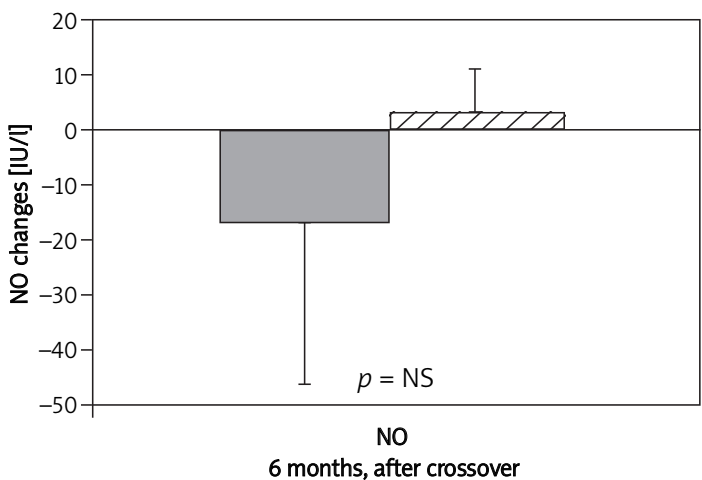

$\square$ FMD decrease $\quad \square \triangle$ FMD increase

Figure 5. FMD changes in relation to $\mathrm{NO}$ and $\mathrm{ET}-1$ concentrations in group $\mathrm{B}^{\star}$ patients (all $p=\mathrm{NS}$ )

FMD - flow-mediated dilation, NO - nitric oxide, ET-1 - endothelin-1

endothelium-dependent vasorelaxation of the brachial artery, owing to increased blood flow [7, 11]. We showed the validity of the measurement of endothelial dysfunction using the FMD approach in a selected group of normolipidaemic, treated hypertensive patients.
Because endothelial dysfunction plays a significant role in the pathogenesis of arterial hypertension, it is reasonable to search for new strategies aimed at improving endothelial function [12]. Several studies indicate that statins could influence blood pressure control in various hypertensive 
patients [5]. This is a pleiotropic statin effect, i.e. independent of lipid-lowering [13].

Still, the hypotensive effect of statin seems to be ambiguous [13]. Koh et al. in their review article interpret various animal and clinical studies regarding the blood pressure lowering statin effect. The variety of results could be related to various blood pressure measurement techniques and various statins used, small sample size study populations, confounding effects of concomitant anti-hypertensive therapy, various criteria, different comparative groups and, finally, various populations studied. It has been observed that statins lower blood pressure values in patients with elevated, but not normal blood pressure regardless of cholesterol level [13]. These results have been confirmed by Borghi et al. in the Brisighella Heart Study - a large randomized controlled trial [14]. Among 1356 patients with hypercholesterolaemia, a significant decrease in blood pressure was observed in the two upper quartiles of systolic blood pressure ( $\geq 140 \mathrm{mmHg}$ ) and was greater in patients treated with statins. Thus, it has been suggested that statins significantly lower blood pressure in patients with uncontrolled hypertension, but not in patients with controlled hypertension or normotension [13]. Moreover, in the CAFE-LLA (The Conduit Artery Function EvaluationLipid-Lowering Arm) - a substudy of an Anglo-Scandinavian Cardiac Outcomes Trial (ASCOT) - 891 patients with hypertension were randomized to atorvastatin $10 \mathrm{mg}$ daily or placebo. Statin therapy did not influence central aortic blood pressure or haemodynamics [15]. The results of PHYLLIS (Plaque Hypertension Lipid-Lowering Italian Study) - a randomized double blind trial - confirmed that the addition of pravastatin to anti-hypertensive treatment did not cause a reduction in systolic and diastolic blood pressure values in patients with low blood pressure at baseline (clinic systolic $160 \mathrm{mmHg}$, 24-h systolic $137 \mathrm{mmHg}$; clinic diastolic $98 \mathrm{mmHg}$ and 24-h diastolic $85 \mathrm{mmHg}$ ) [16].

However, in the previous study, we confirmed the hypotensive effect of atorvastatin in normolipidaemic patients with already treated and well-controlled essential arterial hypertension. What is more, we investigated the influence of statin therapy on various biomarkers which are involved in the pathogenesis of essential arterial hypertension [6]. We discovered that in statin-treated patients the decrease in blood pressure was correlated with the increase in $\mathrm{NO}$ and the decrease in autoantibodies against oxidized LDL (ox-LDL).

In the current study, we aimed to investigate the influence of statin treatment on FMD and intended to assess what the blood pressure would be after stopping atorvastatin therapy. A high dose of atorvastatin decreased blood pressure values and a greater reduction was concurrent with FMD improvement. Conversely, after suspending statin therapy, systolic blood pressure values significantly increased, which was consistent with FMD impairment. Bellia and associates showed that simvastatin significantly improved FMD to a greater extent than rosuvastatin. However, no association between FMD improvement, low-density lipoprotein cholesterol ( $\mathrm{LDL}$ ) reduction, and changes in high-sensitivity C-reactive protein (hs-CRP) levels were found [17]. In our study, we assessed biomarkers of oxidative stress, antioxidative status and endothelial dysfunction, and failed to demonstrate significant relations between them and FMD variation. Based on recent data, we presumed to intensify the investigations. We wanted to find the link between the hypotensive effect of statin, FMD improvement, and changes in concentrations of biomarkers in essential hypertensive patients.

A reduction of $\mathrm{NO}$ and an increase in ET-1 secretions have been observed in hypertensive patients $[2,18]$. Moreover, several diseases, including hypertension, have been proved to be related to oxidative stress and production of free radicals. It has been suggested that high blood pressure is a state associated with a loss of the balance between prooxidation and antioxidation [3]. Increased levels of peroxides and decreases in antioxidants in patients with essential arterial hypertension have been reported earlier [18]. However, to our knowledge, changes in these agents in the aspect of FMD improvement or impairment after statin therapy have not been investigated previously. In our patients, FMD improvement was related to a trend towards increase in total antioxidant status, which was less evident, but still present, after the withdrawal of statin therapy (in patients with FMD impairment). This may suggest a lasting antioxidant statin effect.

Simultaneously, FMD improvement was related to the decrease in peroxide concentration (measured by Oxystat) and FMD impairment was related to its increase. In the same way, NO and ET-1 concentration changes were observed: vasodilatative NO tended to increase and vasoconstrictive ET-1 to decrease after statin therapy, and these effects were consistent with FMD improvement.

We chose a selected group of treated, normolipidaemic patients and added to that treatment a high dose of atorvastatin. There are some data proving that a low dose of atorvastatin could have pleiotropic effects. Kuryata and Yegorova demonstrated an improvement in endothelial function assessed by FMD in patients with coronary artery disease treated with atorvastatin $10 \mathrm{mg}$ daily for 12 weeks [19]. Administration of a statin in hypertensive patients with blood pressure values effectively reduced by traditional anti-hypertensive agents does not have an additional blood pressure 
lowering effect [13]. Thus, we wanted to use a higher dose of atorvastatin to have a better potential effect in treated hypertensives.

Some studies found no change in blood pressure levels with additional statin treatment in patients with controlled hypertension by angiotensin-converting enzyme inhibitors and calcium antagonists or atenolol. The addition of simvastatin to losartan did not enhance the blood pressure lowering action of losartan in hypertensive and hypercholesterolaemic patients [20]. The antihypertensive effect of captopril and atenolol was not influenced by concurrent administration of pravastatin [21]. In hypertensive, hypercholesterolaemic patients, pravastatin remains efficacious as a lipid-lowering agent in the presence of antihypertensive therapy (angiotensin-converting enzyme inhibitors and calcium antagonists), but does not enhance the blood pressure lowering action of these drugs [22]. However, the results of one study demonstrate that the use of statins in combination with anti-hypertensive drugs can improve blood pressure control in patients with uncontrolled hypertension and high serum cholesterol levels [23]. The changes in blood pressure values in our studied hypertensive patients with previous optimal anti-hypertensive treatment and with a normal lipid profile after 3 months of high-dose atorvastatin treatment could be explained by such a synergy with other drugs. Ge et al. have shown that atorvastatin added to amlodipine, apart from inhibition of the inflammatory process and reduction of left ventricular hypertrophy, markedly decreased systolic and diastolic blood pressure values as compared to amlodipine alone [24].

Our hypertensive patients, besides well-controlled diseases, were normolipidaemic. In the previous report, we revealed that the hypotensive effect of atorvastatin in properly treated patients is independent of lipid-lowering [6]. To avoid methodological limitations, a selected group of homogeneous patients was studied. The study was prospective, randomised and had a cross-over design. To assume that both groups of patients received a comparable proportion of anti-hypertensive agents, group B was created to prove the potential hypotensive effect of atorvastatin in group A. Also, in contrast to previous studies, we used ABPM. Available data are largely limited to blood pressure values measured in the clinic without providing information on ambulatory blood pressure, which is of greater prognostic importance [25-27]. Finally, we examined young hypertensive patients, and available data provide information that, at least in patients with metabolic syndrome, FMD changes were observed in the elderly [28].

In conclusion, the present data demonstrate that the hypotensive effect of atorvastatin is related to
FMD improvement in normolipidaemic hypertensive patients with previously diagnosed and treated disease. Thus, there may be a link between atorvastatin action and improvement in endothelial function.

The main limitation of this study is the small number of patients. This might be the reason for trends - not significant changes - in some of the parameters assessed. However, there are studies assuming the same issue, but investigating even smaller groups of patients [29]. Since higher baseline systolic and diastolic blood pressure values could also influence the results, it is hard to compare these studies. Patients with high blood pressure levels at baseline as well as those treated with angiotensin-converting enzyme inhibitors and calcium channel blockers are expected to benefit more in this regard [30]. Furthermore, the differences in baseline levels of peroxides, endothelin and NO between the two groups were quite big; this could have also influenced the results. Perhaps it would be more interesting to compare different doses of the same statin, or different statins (particularly those commonly used - simvastatin, atorvastatin and rosuvastatin). However, our study sought to examine a selected group of hypertensive patients. Moreover, we used a prospective cross-over design to increase the statistical power of the study. Treatment with statins during the studies that reported blood pressure data ranged from 1 to 12 months. A short time of statin treatment could probably influence biochemical results. However, the patients were followed prospectively and were investigated thoroughly; therefore, the authors consider the obtained results as being representative.

\section{Acknowledgments}

This work was supported by The State Committee for Scientific Research (grant No.: N 402075 $31 / 2290$ ). Karol Kaminski is supported by a grant from the Foundation for Polish Science.

\section{References}

1. Vanhoutte PM. Endothelial dysfunction: the first step toward coronary arteriosclerosis. Circ J 2009; 73: 595-601.

2. Taddei S, Virdis A, Ghiadoni L, Sudano I, Notari M, Salvetti $A$. Vasoconstriction to endogenous endothelin- 1 is increased in the peripheral circulation of patients with essential hypertension. Circulation 1999; 100: 1680-3.

3. Romero-Alvira D, Roche E. High blood pressure, oxygen radicals and antioxidants: etiological relationships. Med Hypothesis 1996; 46: 414-20.

4. Banach M, Mikhailidis DP, Kjeldsen SE, Rysz J. Time for new indications for statins? Med Sci Monit 2009; 15: MS1-5.

5. Borghi C, Veronesi M, Prandin MG, Dormi A, Ambrosioni E. Statins and blood pressure regulation. Curr Hypertens Rep 2001; 3: 281-8.

6. Kuklinska A, Mroczko B, Musial WJ, et al. Influence of atorvastatin on blood pressure control in treated hyperten- 
sive, normolipemic patients - an open, pilot study. Blood Press 2010; 19: 260-6.

7. Corretti MC, Anderson TJ, Benjamin EJ, et al.; International Brachial Artery Reactivity Task Force. Guidelines for the ultrasound assessment of endothelial-dependent flowmediated vasodilation of the brachial artery: a report of the International Brachial Artery Reactivity Task Force. J Am Coll Cardiol 2002; 39: 257-65.

8. Kuklinska A, Mroczko B, Musial WJ, et al. High-sensitivity C-reactive protein and total antioxidant status in patients with essential arterial hypertension and dyslipidemia. Adv Med Sci 2009; 54: 225-32.

9. Kuklinska A, Mroczko B, Musial WJ, et al. Diagnostic biomarkers of essential arterial hypertension: the value of prostacyclin, nitric oxide, oxidized-LDL, and peroxide measurements. Int Heart J 2009; 50: 341-51.

10. Sitia S, Tomasoni L, Atzeni F, et al. From endothelial dysfunction to atherosclerosis. Autoimmun Rev 2010; 9: 830-4.

11. Celermajer DS, Sorensen KE, Gooch VM, et al. Non-invasive detection of endothelial dysfunction in children and adults at risk of atherosclerosis. Lancet 1992; 340: 1111-5.

12. Ast J, Jablecka A, Bogdanski P, Smolarek I, Krauss H, Chmara E. Evaluation of the antihypertensive effect of L-arginine supplementation in patients with mild hypertension assessed with ambulatory blood pressure monitoring. Med Sci Monit 2010; 16: CR266-71.

13. Koh KK, Quon MJ, Waclawiw MA. Are statins effective for simultaneously treating dyslipidemias and hypertension? Atherosclerosis 2008; 196: 1-8.

14. Borghi C, Dormi A, Veronesi M, Sangiorgi Z, Gaddi A. Brisighella Heart Study Working Party Association between different lipid-lowering treatment strategies and blood pressure control in the Brisighella Heart Study. Am Heart J 2004; 148: 285-92.

15. Williams B, Lacy PS, Cruickshank JK, et al.; CAFE and ASCOT Investigators. Impact of statin therapy on central aortic pressures and hemodynamics: principal results of the Conduit Artery Function Evaluation-Lipid-Lowering Arm (CAFE-LLA) Study. Circulation 2009; 119: 53-61.

16. Mancia G, Parati G, Revera M, et al. Statins, antihypertensive treatment, and blood pressure control in clinic and over 24 hours: evidence from PHYLLIS randomised double blind trial. BMJ 2010 25; 340:c1197. doi: 10.1136/bmj.c1197.

17. Bellia A, Rizza S, Galli A, et al. Early vascular and metabolic effects of rosuvastatin compared with simvastatin in patients with type 2 diabetes. Atherosclerosis 2010; 210: 199-201.

18. Kashyap MK, Yadav V, Sherawat BS, et al. Different antioxidants status, total antioxidant power and free radicals in essential hypertension. Mol Cell Biochem 2005; 277: 89-99.

19. Kuryata OV, Yegorova YV. The influence of low-dose atorvastatin on lipid levels and endothelial vascular function in patients with significant coronary artery stenosis. Kardiol Pol 2006; 64: 44-8.

20. Koh KK, Quon MJ, Han SH, et al. Additive beneficial effects of losartan combined with simvastatin in the treatment of hypercholesterolemic, hypertensive patients. Circulation 2004; 110: 3687-92.

21. Foss OP, Graff-Iversen S, Istad H, Søyland E, Tjeldflaat L, Graving B. Treatment of hypertensive and hypercholesterolaemic patients in general practice. The effect of captopril, atenolol and pravastatin combined with life style intervention. Scand J Prim Health Care 1999; 17: 122-7.

22. O'Callaghan CJ, Krum H, Conway EL, et al. Short term effects of pravastatin on blood pressure in hypercholes terolaemic hypertensive patients. Blood Press 1994; 3: 404-6.

23. Borghi C, Prandin MG, Costa FV, et al. Use of statins and blood pressure control in treated hypertensive patients with hypercholesterolemia. J Cardiovasc Pharmacol 2000; 35: 549-55.

24. Ge CJ, Lu SZ, Chen YD, Wu XF, Hu SJ, Ji Y. Synergistic effect of amlodipine and atorvastatin on blood pressure, left ventricular remodeling, and C-reactive protein in hypertensive patients with primary hypercholesterolemia. Heart Vessels 2008; 23: 91-5.

25. Mancia G, Parati G. Guiding antihypertensive treatment decisions using ambulatory blood pressure monitoring. Curr Hypertens Rep 2006; 8: 330-7.

26. O'Brien E, Asmar R, Beilin L, et al.; European Society of Hypertension Working Group on Blood Pressure Monitoring. European Society of Hypertension recommendations for conventional, ambulatory and home blood pressure measurement. J Hypertens 2003; 21: 821-48.

27. Sethi A, Arora RR. Ambulatory blood pressure as a pre dictor of cardiovascular risk. Arch Med Sci 2009; 5: 3-9.

28. de Matthaeis A, Greco A, Serviddio G, Stramaglia G, Vendemiale $G$. Endothelial dysfunction evaluated by flow mediated dilation is strongly associated to metabolic syndrome in the elderly. Aging Clin Exp Res 2009; 22: 303-7.

29. Glorioso N, Troffa C, Filigheddu F, et al. Effect of the HMG-CoA reductase inhibitors on blood pressure in patients with essential hypertension and primary hypercholesterolemia. Hypertension 1999; 34: 1281-6.

30. Kostapanos MS, Milionis HJ, Elisaf MS. Current role of statins in the treatment of essential hypertension. Expert Opin Pharmacother 2010; 11: 2635-50. 